\title{
(6) OPEN ACCESS \\ National surveillance for type 1, type 2 diabetes and prediabetes among children and adolescents: a population-based study (SAUDI-DM)
}

\author{
Khalid Al-Rubeaan
}

- Additional material is published online only. To view please visit the journal online (http://dx.doi.org/10.1136/jech2015-205710).

\section{Correspondence to} Dr Khalid Al-Rubeaan, University Diabetes Center, Collage of Medicine, King Saud University, P.O. Box 18397, Riyadh 11415, Saudi Arabia;

krubeaan@dsrcenter.org

Received 2 March 2015 Revised 12 May 2015 Accepted 2 June 2015 Published Online First 17 June 2015

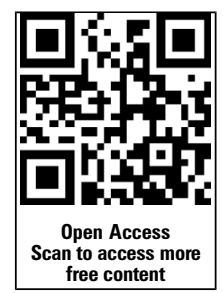

CrossMark

To cite: Al-Rubeaan K. J Epidemiol Community Health 2015;69:1045-1051.

\section{ABSTRACT}

Background There is a paucity of data on the national prevalence of diabetes and prediabetes among youth. The Saudi Abnormal Glucose Metabolism and Diabetes Impact Study (SAUDI-DM) was used to assess the prevalence of type 1 and type 2 diabetes as well as impaired fasting glucose (IFG) among children and adolescents.

Methods Sociodemographic, anthropometric and clinical data were collected through a nationwide household randomly selected 23523 children and adolescents aged $\leq 18$ years. Known participants with diabetes were classified according to their diabetes type, while participants without diabetes were subjected to fasting plasma glucose assessment and patients with diabetes were identified using the American Diabetes Association (ADA) criteria. All the studied participants were tested for lipid parameters. Multivariate logistic regression analysis was used to assess different risk factors.

Results The overall prevalence of diabetes was $10.84 \%$, of which $0.45 \%$ were known type 1 and type 2 patients with diabetes and $10.39 \%$ were either newly identified cases of diabetes $(4.27 \%)$ or IFG $(6.12 \%)$ with more than $90 \%$ of the participants with diabetes being unaware of their disease. The prevalence of known type 1 and type 2 diabetes as well as the newly identified cases was higher than what has been reported internationally. Age, male gender, obesity, urban residency, high family income and presence of dyslipidaemia were found to be significant risk factors for diabetes and IFG.

Conclusions Diabetes and IFG are highly prevalent in this society with the majority of the patients being unaware of their disease, which warrants urgent adoption of early detection, treatment and prevention programmes.

\section{INTRODUCTION}

The emergence of diabetes mellitus as a global public health problem in children and adolescents is due to the widespread obesity and pronounced lifestyle changes. ${ }^{1}{ }^{2}$ In the past, type 1 diabetes used to be the predominant type among children, but for the last 20 years, type 2 diabetes, ${ }^{3}{ }^{4}$ which is known for its different aetiology, is taking the lead. A special focus on identifying the factors behind the aetiology of this new observation, especially in communities known for their high prevalence of type 2 diabetes, would help to understand this epidemiological shift.

There are limited number of community-based studies that have looked into the prevalence of type 1 and type 2 diabetes simultaneously among children and adolescents worldwide. ${ }^{15}$ Between the years 1990 and 2008, the incidence of type 1 diabetes has almost doubled from $2.8 \%$ to $4.0 \%$ per year worldwide, ${ }^{6}$ while that of type 2 diabetes has increased 10 times in young children aged 6-12 years and almost doubled among adolescents, rising from 7.3 to $13.9 / 100000$ between the years 1967 and $1997 . .^{4}$ In the USA, a national data set reported that the prevalence of type 2 diabetes in youth aged 10-19 years had increased by $30.5 \%$ between 2001 and 2009. ${ }^{6}$ Obesity is a well-known risk factor for type 2 diabetes in children and adolescents, which is a consequence of high caloric intake and physical inactivity, in addition to the sharp increase in the use of computer and TV watching. ${ }^{7}$ Ethnicity and genetic susceptibility related to a positive family history have been recognised as predisposing risk factors for type 2 diabetes in addition to prenatal exposure to maternal undernutrition or gestational diabetes. ${ }^{6-10}$

Saudi Arabia, currently ranked seventh among the top 10 countries known for their high prevalence of diabetes globally, sets a good model to study the factors behind the increase in the prevalence of type 2 diabetes among children and adolescents. As a part of the Saudi Abnormal Glucose Metabolism and Diabetes Impact Study (SAUDI-DM), ${ }^{11}$ the current study explores the prevalence of type 1 and type 2 diabetes in addition to impaired fasting glucose (IFG) and their modifiable and non-modifiable risk factors among children and adolescents.

\section{METHODS}

\section{Study design}

SAUDI-DM is a nationwide household populationbased cross-sectional study using a multistage stratified cluster sampling technique taking into consideration the urban to rural ratio in the 13 administrative regions in Saudi Arabia during the period from 2007 to 2009. The study was conducted through primary healthcare centres by trained physicians and nurses through recruiting Saudi nationals' family members from every third house in the selected areas. All family members who were available during the visit of the survey team were recruited regardless of their age, gender or diabetes status, excluding participants who refused to participate or were not present during the recruitment visit.

Using the Saudi national regional census survey in 2007, 87417 participants were recruited and adjusted for age, area of residency and gender distribution excluding 34047 participants. Of the 
remaining 53370 adjusted participants, children and adolescents aged $\leq 18$ years totalling to 23523 participants were considered the eligible study cohort.

All demographic data which include participants' age, gender and family history of diabetes were collected by a standard questionnaire. The area of residency was classified according to the Ministry of Municipal and Rural Affairs classification and the family's monthly income was expressed in Saudi Riyals (SR) $(1 \mathrm{SR}=\mathrm{US} \$ 0.267)$. Any participant known to have diabetes was classified based on the patient's age at diagnosis, history of ketocidosis, and management into either type 1 if the patient was diagnosed before 30 years of age and was using insulin with or without a history of diabetic ketoacidosis, or type 2 if the patients was diagnosed after the age of 30, having an elevated blood glucose which is currently being managed with diet alone or with oral hypoglycaemic agents with or without insulin and with no history of diabetic ketoacidosis. A total of 72 participants who were not sure about their diabetic status and were not on any treatment or not being followed clinically were considered non-diabetic and were subjected to screening using fasting plasma glucose (FPG).

Anthropometric measurements of weight and height were used to calculate body mass index (BMI) for age-specific and gender-specific percentile, where overweight was considered when BMI was from $\geq 85$ th to $<95$ th centile and obesity was considered when BMI was $\geq 95$ th centile. ${ }^{12}$ At the same time, systolic and diastolic blood pressure (DBP) were measured in a sitting position using a mercury sphygmomanometer with appropriate cuff size.

Since children $<6$ years of age have difficulty in fasting more than $10 \mathrm{~h}$ overnight and may not be capable of providing a venous blood sample, a total of 6211 (26.52\%) children $<6$ years were not included for FPG assessment. All participants with and without diabetes aged $\geq 6$ years, in addition to children with diabetes aged $<6$ years, were asked to report to the allocated primary care centres for venous blood collection after $10 \mathrm{~h}$ of overnight fasting with the usual activity and diet. The overall response rate for FPG collection was $86.39 \%$.

Blood glucose, serum cholesterol, high-density lipoprotein (HDL), low-density lipoprotein (LDL) and triglyceride assessments were performed at the Strategic Center for Diabetes research laboratory using the Mindray (B5-200) chemistry analyser.

This study was approved by the Institutional Review Board (IRB) at the College of Medicine, King Saud University. All children and adolescents who were participating in this study consented either through their legal guardian or directly if they were older than 14 years, which is the legal age in the Kingdom.

\section{Case identification}

According to the American Diabetes Association (ADA) criteria $2003,{ }^{13}$ FPG is normal if the level is $<110 \mathrm{mg} / \mathrm{dL}(<6.1 \mathrm{mmol} / \mathrm{L})$ and is considered IFG if the level is between $110 \mathrm{mg} / \mathrm{dL}$ $(6.1 \mathrm{mmol} / \mathrm{L})$ and $125 \mathrm{mg} / \mathrm{dL}(6.9 \mathrm{mmol} / \mathrm{L})$, while diabetes is considered when FPG is $\geq 126 \mathrm{mg} / \mathrm{dL}(7.0 \mathrm{mmol} / \mathrm{L})$.

Age-specific and gender-specific 85 th centile values of systolic blood pressure (SBP) and DBP were used to define high blood pressure among the study participants. Using the National Cholesterol Education programme (NCEP) guidelines, ${ }^{14}$ triglycerides (TR) were considered high if the level was $\geq 1.13 \mathrm{mmol} / \mathrm{L}$ for participants $\leq 9$ years and $1.47 \mathrm{mmol} / \mathrm{L}$ for participants aged 10-18 years. LDL cholesterol was considered high if the level was $\geq 3.37 \mathrm{mmol} / \mathrm{L}$, while total cholesterol was considered high if the level was $\geq 5.18 \mathrm{mmol} / \mathrm{L}$. HDL was considered low if the level was $\leq 0.91 \mathrm{mmol} / \mathrm{L}$.

\section{Statistical analysis}

The strengthening reporting of observational study in epidemiology (STROBE) guidelines were used to design and report this study. All data were analysed using Software Package for Social Sciences (SPSS) V.20.0. A t test was used for continuous data, and $\chi^{2}$ test for categorical ones. Overweight and obesity were defined using sex-specific and age-specific between 85 th and 95th centiles. The same was used for both systolic and DBP to define high blood pressure at the 85 th centile. Imputation, based on a regression model, was used to estimate missing data. The prevalence of diabetes and IFG was estimated for the whole study population and for population subgroups taking into account the sampling weight. Pearson's correlation (r) was used to assess the strength of association between the age and the prevalence of known type 1 , type 2 and new diabetes and IFG cases. Factors that were known as being significantly associated with childhood diabetes were assessed using univariate, age-adjusted and gender-adjusted, and multivariate logistic regression models. OR and its 95\% CIs were used to express different risks. A p value of $<0.05$ was chosen as the level of significance.

\section{RESULTS}

\section{Diabetes and IFG prevalence}

Out of the total studied sample, 105 participants $(0.45 \%)$ were found to be known children with diabetes or adolescents aged $\leq 18$ years, of which $88(0.38 \%)$ were type 1 and $17(0.07 \%)$ were type 2 patients with diabetes. Of 17207 (73.48\%) participants aged 6-18 years who were tested for FPG, 1053 (6.12\%) were IFG cases, and 735 (4.27\%) were newly identified cases with diabetes totalling $10.39 \%$ (figure 1 ).

Known type 1 diabetes age-specific prevalence showed an increasing trend, starting from 2.5/1000 at birth and increasing to $4.6 / 1000$ at 18 years of age with $r=0.432$. On the other hand, known type 2 diabetes age-specific prevalence showed a flat rate at 1/1000 for all age groups with $r=0.089$ (figure 2A). The age-specific prevalence of the newly discovered cases with diabetes showed a horizontal trend at the rate of 45/1000 from 6 to 18 years of age with $r=0.093$, while it showed a significant increasing trend with $\mathrm{r}=0.628$, starting from 58 cases and reaching up to 68 cases per 1000 at the age of 18 years for IFG cases (figure 2B).

\section{Clinical and biochemical characteristics}

In the studied cohort, children and adolescents with diabetes were always older and the mean age was significantly higher in known type 2 patients with diabetes and IFG cases. The mean BMI and waist circumference were the highest among known type 2 patients with diabetes, while both SBP and DBP were the highest among known type 1 patients with diabetes. The total cholesterol, LDL and TR were found to be high in all diabetic groups, but were significant in the newly identified cases with diabetes and IFG as shown in table 1.

It can be observed from table 2 that the frequency of different types of diabetes increased with age, reaching its peak in the age group between 13 and 18 years. There were more females in the known cases with diabetes and more males in the newly identified cases with diabetes and IFG. A positive family history of diabetes was frequent in the known cases with type 1 , type 2 , newly identified diabetes and IFG. The majority of the cases with diabetes and IFG were living in urban areas and had a family 


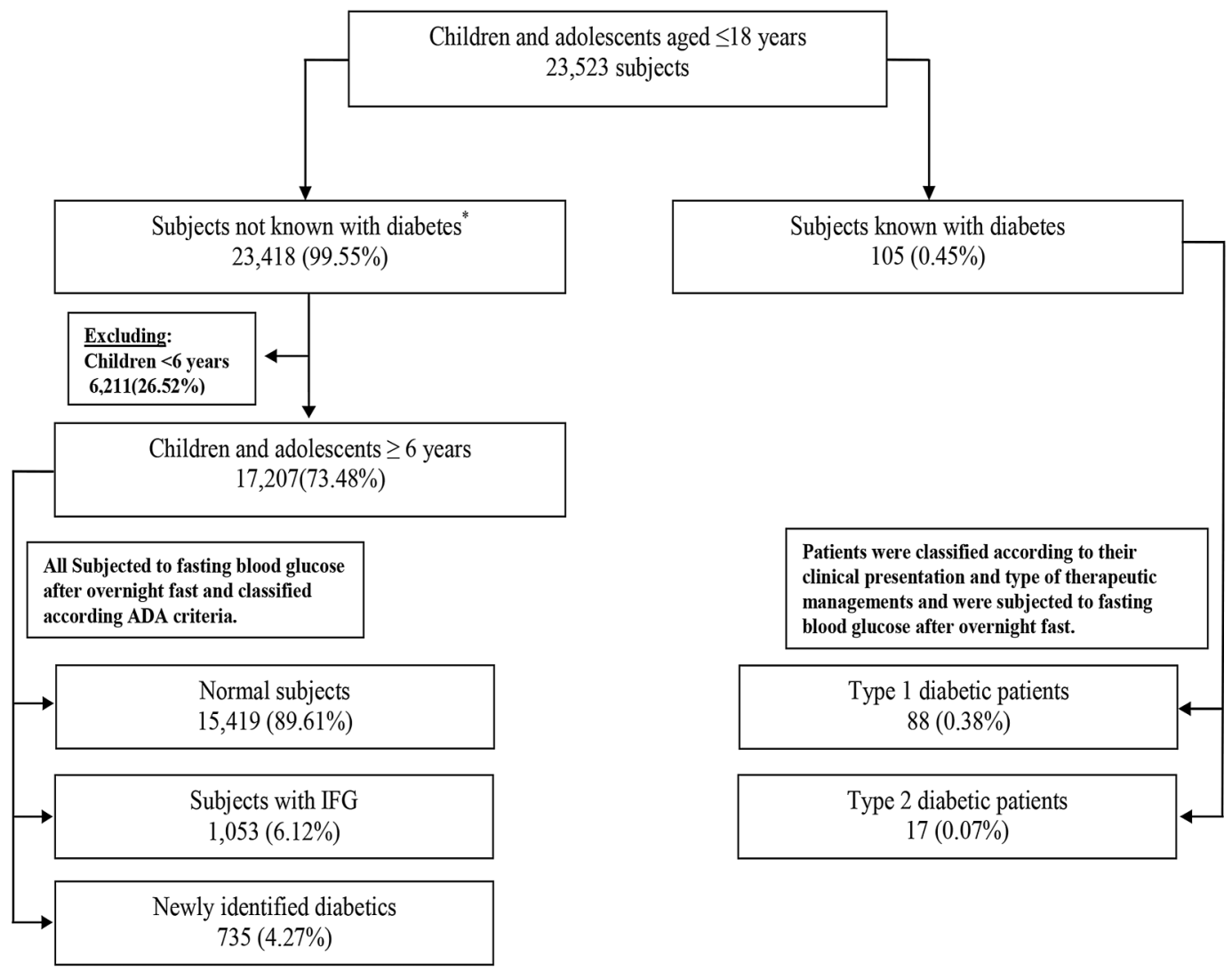

* 72 subjects were unconfirmed diabetic children and adolescents and were not on any treatment or being followed clinically, so they were considered non diabetic and subjected for screening with fasting plasma glucose.

Figure 1 Flow diagram for the studied cohort of children and adolescents (0-18 years) in relation to abnormal glucose metabolism status.

monthly income $<8000 \mathrm{SR}$. Obesity frequencies expressed by BMI $\geq 95$ th centile were $18.18 \%, 23.53 \%, 15.51 \%$ and $20.65 \%$ for cases with type 1, type 2, newly identified diabetes and IFG, respectively. Diastolic hypertension was more prevalent than systolic hypertension in the known cases with type 1 and type 2 diabetes and newly identified and IFG cases. The frequency of elevated cholesterol was the highest among the newly identified cases with diabetes and IFG at $43.13 \%$ and $31.37 \%$, respectively, while the TRs were the highest among new cases identified with diabetes and known type 1 patients with diabetes at $50.34 \%$ and $40.91 \%$, respectively.

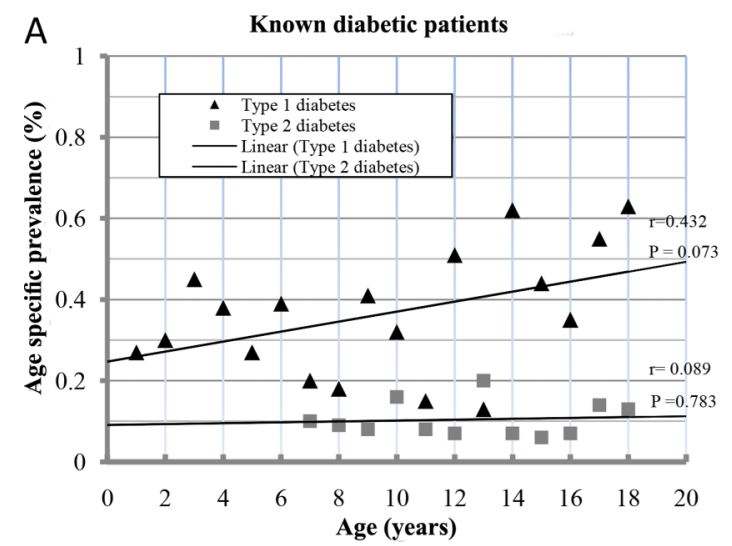

\section{Risk factors}

Table 3 shows the risk factors associated with the newly identified cases with diabetes and IFG aged between 6 and 18 years. Age $\geq 13$ years was a significant risk factor, as shown from univariate analysis, and an independent risk, as calculated by the multivariate model. Male gender was a significant risk factor in the univariate but not in the multivariate logistic regression analysis. Both urban residency and family income more than 8000 SR were significant in the univariate, age-adjusted and genderadjusted and multivariate analyses, while BMI $\geq 95$ th centile was found to be a significant risk only in the univariate and

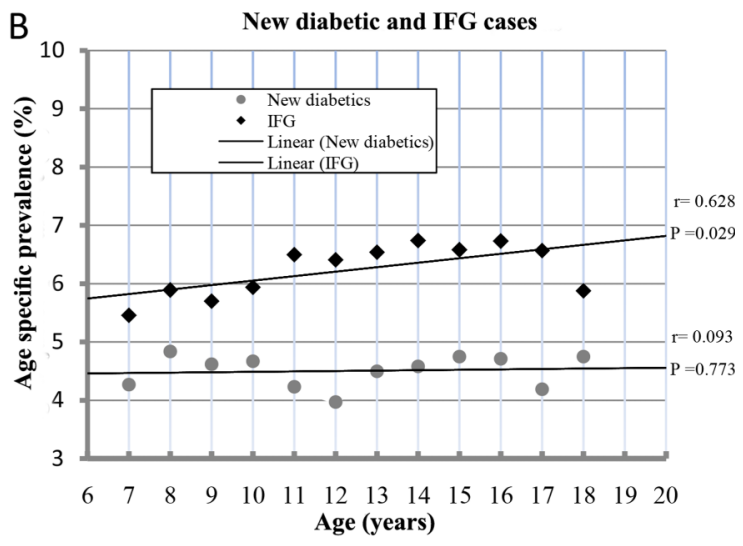

Figure 2 The age-specific prevalence using simple linear regression analysis for the known type 1 and type 2 diabetic patients (A) and the newly diagnosed diabetic and impaired fasting glucose (IFG) cases (B). 


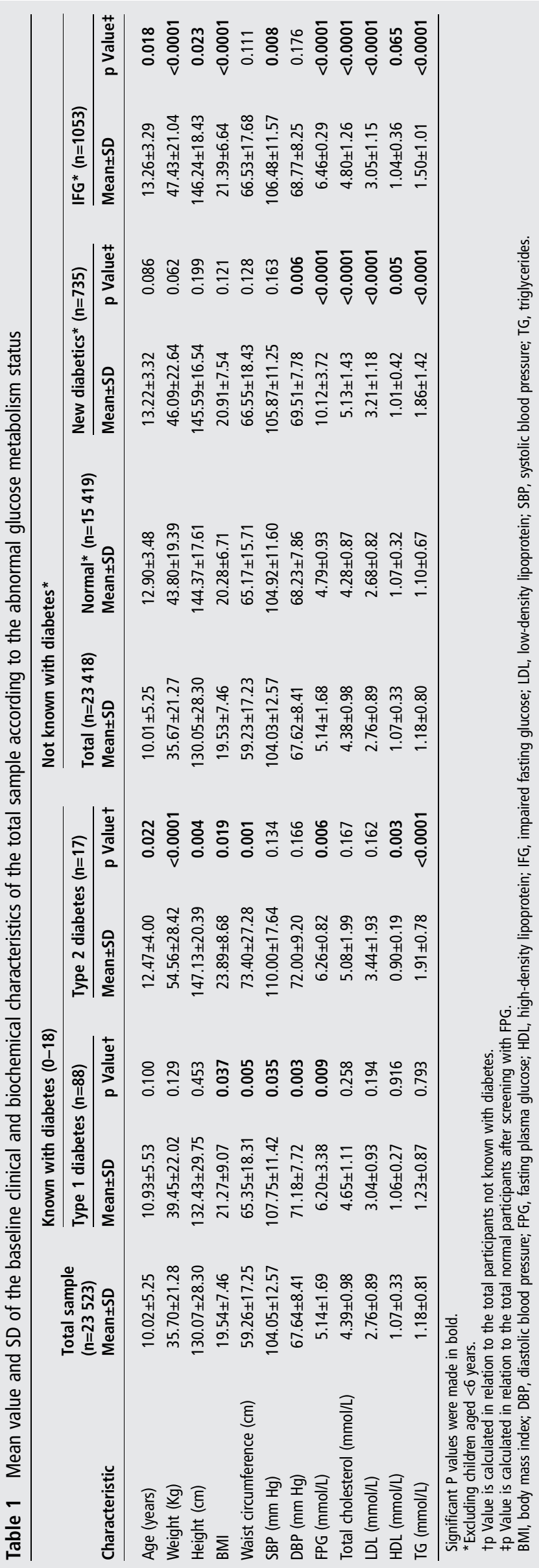

age-adjusted and gender-adjusted regression analyses. High total cholesterol and TRs and low HDL were significant risk factors in univariate, age-adjusted and gender-adjusted and multivariate analyses.

\section{DISCUSSION}

This study reported the prevalence of diabetes to be $10.83 \%$ among children and adolescents aged $\leq 18$ years, which is one of the highest reported prevalences in this age group and is in line with the fact that Saudi Arabia is among the top 10 countries with a high prevalence of diabetes. The other striking revelation from this study was that more than $90 \%$ of the children and adolescents with diabetes were unaware of their disease, of which the known patients with type 1 and type 2 diabetes accounted for only $0.45 \%$, while the newly identified cases with diabetes and IFG accounted for $10.39 \%$. These hidden cases mandate early screening programmes for IFG and diabetes particularly in obese children and adolescents. Since medical literature has shown that lifestyle intervention to reduce obesity in adolescents to prevent type 2 diabetes is too late, it is recommended to prevent childhood overweight, especially when it is known that obesity is very challenging once it is established. ${ }^{15}$

\section{Known patients with diabetes}

The prevalence of known type 1 and type 2 diabetes in our study is double of what had been reported earlier from a multiethnic SEARCH study cohort aged $\leq 19$ years. $^{6}$ When comparing our findings for the age group between 12 and 18 years with the National Health and Nutrition Examination Survey (NHANS), ${ }^{16}$ the prevalence of type 1 diabetes was almost similar, while that of known type 2 diabetes, which is considered to be a silent disease, was less prevalent in our cohort and could be a reflection of underdiagnosis. This was also proved by our findings that the prevalence of newly identified cases with diabetes for the same age group was high at $4.57 \%$ (see online supplementary table $\mathrm{S} 1$ ).

The trend for age-specific prevalence for the previously identified type 1 participants with diabetes was increasing with age, while it was not the case for the previously identified type 2 participants with diabetes. This could indicate that the age effect is more pronounced in type 1 , while it is unexpectedly not the case for type 2 . This could be due to the small number of known cases with type 2 diabetes or the effect of the large number of patients with undiagnosed IFG and type 2 diabetes, which has an increasing pattern with age as shown in figure $2 \mathrm{~B}$.

\section{Newly identified cases}

The prevalence of newly identified cases with diabetes from this study for the age group between 6 and 18 years was four times higher than what had been reported earlier among Native Americans in 2010 at $1.0 \%$, while being similar for cases with IFG. ${ }^{17}$ However, when the prevalence rate of new cases with diabetes aged 12-18 years in our cohort was compared with that for the same age group from a Canadian study, the prevalence rate in our study was $4.57 \%$ versus $3.5 \%$ in that Canadian study. ${ }^{5}$ The prevalence of IFG in this specific age group reported by the NHANS study was two times more than that observed in our study at $13.1 \%$ vs $6.62 \% .{ }^{18}$ This could be explained by the lower cut-off value of $100 \mathrm{mg} / \mathrm{dL}$ used to identify cases with IFG in the NHANS study compared to our cut-off value of $110 \mathrm{mg} / \mathrm{dL}$.

The IFG to diabetes ratio of the newly identified cases in our study was 1.5 , which is similar to what has been reported by the aboriginal Canadian community study. ${ }^{19}$ This observation 
Table 2 The frequency analysis of clinical and biochemical markers for the total sample and according to the abnormal glucose metabolism status

\begin{tabular}{|c|c|c|c|c|c|c|c|}
\hline \multirow[b]{2}{*}{ Characteristic } & \multirow[b]{2}{*}{ Total sample } & \multicolumn{2}{|c|}{ Known with diabetes } & \multicolumn{4}{|c|}{ Not known with diabetes } \\
\hline & & Type 1 & Type 2 & Total & Normal* & New diabetics* & IFG* \\
\hline Number & 23523 & 88 & 17 & 23418 & 15419 & 735 & 1053 \\
\hline \multicolumn{8}{|l|}{ Age (years) } \\
\hline$<6$ & $6232(26.49)$ & $21(23.86)$ & 0 & $6211(26.52)$ & 0 & 0 & 0 \\
\hline $6-12$ & $8259(35.11)$ & $26(29.55)$ & $8(47.06)$ & $8225(35.12)$ & $6714(43.54)$ & $290(39.46)$ & $408(38.75)$ \\
\hline $13-18$ & $9032(38.40)$ & $41(46.59)$ & $9(52.94)$ & $8982(38.36)$ & $8705(56.46)$ & $445(60.54)$ & $645(61.25)$ \\
\hline \multicolumn{8}{|l|}{ Gender } \\
\hline Male & $11708(49.77)$ & $39(44.32)$ & $8(47.06)$ & $11661(49.80)$ & $7606(49.33)$ & $391(53.20)$ & $552(52.45)$ \\
\hline Female & $11815(50.23)$ & $49(55.68)$ & $9(52.94)$ & $11757(50.20)$ & $7813(50.67)$ & $344(46.80)$ & $501(47.55)$ \\
\hline \multicolumn{8}{|c|}{ Family history of diabetes } \\
\hline Yes & $12218(51.94)$ & $67(76.14)$ & $12(70.59)$ & $12139(51.84)$ & $8544(55.41)$ & $385(52.38)$ & $539(51.19)$ \\
\hline \multicolumn{8}{|l|}{ Residency } \\
\hline Urban & 17339 (73.71) & $68(77.27)$ & $14(82.35)$ & $17257(73.69)$ & $10068(65.30)$ & 499 (67.89) & $779(73.97)$ \\
\hline Rural & $6184(26.29)$ & $20(22.73)$ & $3(17.65)$ & $6161(26.31)$ & $5351(34.70)$ & $236(32.11)$ & $274(26.03)$ \\
\hline \multicolumn{8}{|l|}{ Income } \\
\hline$<8000$ SR & $17773(75.55)$ & $60(68.18)$ & $12(70.59)$ & 17701 (75.59) & $12178(78.98)$ & $504(68.57)$ & $797(75.73)$ \\
\hline$\geq 8000$ SR & $5750(24.45)$ & $28(31.82)$ & $5(29.41)$ & $5717(24.41)$ & $3241(21.02)$ & $231(31.43)$ & $256(24.27)$ \\
\hline \multicolumn{8}{|l|}{ Body mass index } \\
\hline$<95$ th centile & $19712(83.80)$ & $72(81.82)$ & $13(76.47)$ & $19627(83.81)$ & $13269(86.06)$ & $621(84.49)$ & $836(79.35)$ \\
\hline$\geq 95$ th centile & $3811(16.20)$ & $16(18.18)$ & $4(23.53)$ & 3791 (16.19) & $2150(13.94)$ & $114(15.51)$ & $217(20.65)$ \\
\hline \multicolumn{8}{|c|}{ Systolic blood pressure } \\
\hline$<85$ th centile & $19879(84.51)$ & $70(79.55)$ & $14(82.35)$ & $19795(8453)$ & $13456(87.27)$ & $644(87.62)$ & $884(83.94)$ \\
\hline$\geq 85$ th centile & 3644 (15.49) & $18(20.45)$ & $3(17.65)$ & $3623(15.47)$ & $1963(12.73)$ & $91(12.38)$ & $169(16.06)$ \\
\hline \multicolumn{8}{|c|}{ Diastolic blood pressure } \\
\hline$<85$ th centile & 17751 (75.46) & $63(71.59)$ & $13(76.47)$ & 17675 ( 75.48) & $12400(80.42)$ & $555(75.51)$ & $822(78.10)$ \\
\hline$\geq 85$ th centile & $5772(24.54)$ & $25(28.41)$ & $4(23.53)$ & $5743(24.52)$ & 3019 (19.58) & $180(24.49)$ & $231(21.90)$ \\
\hline \multicolumn{8}{|c|}{ Total cholesterol $(\mathrm{mmol} / \mathrm{L})$} \\
\hline$<5.18$ & $19559(83.15)$ & $62(70.45)$ & $12(70.59)$ & $19485(83.21)$ & $13391(86.85)$ & $418(56.87)$ & $723(68.63)$ \\
\hline$\geq 5.18$ & $3964(16.85)$ & $26(29.55)$ & $5(29.41)$ & $3933(16.79)$ & $2028(13.15)$ & $317(43.13)$ & $330(31.37)$ \\
\hline \multicolumn{8}{|c|}{ Low-density lipoprotein (mmol/L) } \\
\hline$<3.37$ & $18818(80.00)$ & $52(59.09)$ & $10(58.82)$ & $18756(80.09)$ & $12828(83.20)$ & $436(59.32)$ & $733(69.63)$ \\
\hline$\geq 3.37$ & $4705(20.00)$ & $36(40.91)$ & $7(41.18)$ & $4662(19.91)$ & $2591(16.80)$ & $299(40.68)$ & $320(30.37)$ \\
\hline \multicolumn{8}{|c|}{ High-density lipoprotein (mmol/L) } \\
\hline$<0.91$ & $7313(31.09)$ & $21(23.86)$ & $6(35.29)$ & $7286(31.11)$ & $4712(30.56)$ & $322(43.81)$ & $385(36.59)$ \\
\hline$\geq 0.91$ & $16210(68.91)$ & $67(76.14)$ & $11(64.71)$ & $16132(68.89)$ & $10707(69.44)$ & $413(56.19)$ & $668(63.41)$ \\
\hline \multicolumn{8}{|c|}{ Triglycerides (mmol/L) } \\
\hline$<1.13+<1.47 \dagger$ & $17558(74.64)$ & $52(59.19)$ & $12(70.59)$ & $17494(74.70)$ & $12249(79.44)$ & $365(49.66)$ & $645(61.26)$ \\
\hline$\geq 1.13+\geq 1.47 \dagger$ & 5965 (25.36) & $36(40.91)$ & $5(29.41)$ & $5924(25.30)$ & $3170(20.56)$ & $370(50.34)$ & $408(38.74)$ \\
\hline
\end{tabular}

${ }^{*}$ Excluding children $<6$ years of age.

tAccording to the NCEP where triglycerides were considered high if the level was $\geq 1.13 \mathrm{mmol} / \mathrm{L}$ for participants $\leq 9$ years and $1.47 \mathrm{mmol} / \mathrm{L}$ for participants aged $10-18$ years.

IFG, impaired fasting glucose; NCEP, National Cholesterol Education programme; SR, Saudi Riyals.

remains true when looking at the age-specific diabetes and IFG prevalence trend.

\section{Risk factors}

This study analysed the different risk factors for newly discovered abnormal glucose metabolism cases, which are most likely cases with type 2 and IFG. Age $\geq 13$ years demonstrated a significant risk factor for abnormal glucose metabolism in our cohort, similar to what had been observed earlier in a Taiwanese study. ${ }^{20}$ This could be supported by the growing evidence that puberty is associated with increased insulin resistance as proved by other epidemiological studies when looking for hyperinsulinaemia in pubertal-age adolescents. ${ }^{21}$ In this study, male gender was also found to be a significant risk factor for this age group. This finding contradicts the observation in other ethnicities like Taiwanese, Native American and Jamaican, ${ }^{20} 2223$ which could either be the result of ethnicity effect or the significantly increased prevalence of obesity among males in our cohort. In addition, the risk calculation in our study included adding IFG to cases with diabetes which may have made the male gender more pronounced since the risk of IFG is more among males in different ethnic groups including our cohort. ${ }^{18} 24$

Similar to what had earlier been reported in other ethnicities, BMI higher than 85 th centile was found to be a significant risk factor for abnormal glucose metabolism in this cohort. Surprisingly, a family history of diabetes was not found to be a significant risk factor, contradicting what was already known in the literature. ${ }^{25}$ This could be explained by the high rate of positive family history that exceeded $30 \%$ and the high rate of consanguineous marriage reaching up to $42 \%$ in our society. ${ }^{26}$

Living in urban area and having monthly family income $\geq 8000$ SR, are significant risk factors for diabetes and IFG 
Table 3 Univariate, age-adjusted and gender-adjusted and multivariate analysis with OR and 95\% Cls for risk factors of the newly diagnosed diabetic and IFG cases

\begin{tabular}{|c|c|c|c|c|c|c|c|c|c|}
\hline \multirow[b]{2}{*}{ Risk factors } & \multicolumn{3}{|c|}{ Univariate } & \multicolumn{3}{|c|}{ Age and gender adjusted } & \multicolumn{3}{|c|}{ Multivariate adjusted } \\
\hline & OR & $95 \% \mathrm{Cl}$ & p Value & OR & $95 \% \mathrm{Cl}$ & p Value & OR & $95 \% \mathrm{Cl}$ & p Value \\
\hline Age $\geq 13$ years & 1.34 & 1.17 to 1.54 & $<0.0001$ & - & - & - & 1.46 & 1.23 to 1.74 & $<0.0001$ \\
\hline Male gender & 1.14 & 1.03 to 1.25 & 0.011 & - & - & - & 1.17 & 0.99 to 1.39 & 0.74 \\
\hline Urban residency & 1.34 & 1.20 to 1.49 & $<0.0001$ & 1.33 & 1.15 to 1.55 & $<0.0001$ & 1.58 & 1.30 to 1.92 & $<0.0001$ \\
\hline Family income $>8000$ SR & 1.42 & 1.27 to 1.59 & $<0.0001$ & 1.41 & 1.18 to 1.70 & $<0.0001$ & 1.36 & 1.12 to 1.65 & 0.002 \\
\hline Body mass index $\geq 95$ centile & 1.34 & 1.12 to 1.61 & 0.002 & 1.32 & 1.10 to 1.59 & 0.003 & 0.97 & 0.77 to 1.22 & 0.788 \\
\hline High cholesterol* & 3.75 & 3.36 to 4.17 & $<0.0001$ & 3.69 & 3.18 to 4.29 & $<0.0001$ & 3.34 & 2.76 to 4.05 & $<0.0001$ \\
\hline High triglycerides $†$ & 2.98 & 2.69 to 3.29 & $<0.0001$ & 2.94 & 2.55 to 3.39 & $<0.0001$ & 2.06 & 1.71 to 2.49 & $<0.0001$ \\
\hline Low high-density lipoprotein & 1.49 & 1.34 to 1.64 & $<0.0001$ & 1.47 & 1.28 to 1.69 & $<0.0001$ & 1.20 & 0.99 to 1.45 & 0.051 \\
\hline
\end{tabular}

${ }^{*}$ High cholesterol $\geq 5.18 \mathrm{mmol} / \mathrm{L}$.

tHigh triglycerides when the level is $\geq 1.3 \mathrm{mmo} / \mathrm{L}$ in children aged $6-9$ years and $\geq 1.47$ in children aged $10-18$ years

fLow HDL if the level is $<0.9 \mathrm{mmo} / \mathrm{L}$.

$\mathrm{HDL}$, high-density lipoprotein;

IFG, impaired fasting glucose; SR, Saudi Riyals.

which is similar to what has earlier been observed in Bangladesh. $^{27}$

Similar to the observation in other ethnicities, ${ }^{28}$ the abnormal lipids in the form of high cholesterol and TRs or low HDL were significant and independent risk factors. Since the abnormal lipid parameters and obesity are part of the metabolic syndrome, which has been recognised more recently among children and adolescents, this could explain the significant high OR observed in this cohort.

This study draws its strength from having a large random sample of children and adolescents in a country known to have a diabetes epidemic. This study was limited by using FPG for defining cases with diabetes, even though it is one of the recommended criteria for diabetes and IFG screening. Another limitation of this study was the possibility that the enrolled participants might have not adhered to the instructions of strict overnight fasting, in addition to excluding children between 0 and 6 years from FPG screening, although this was justified in the methodology section. We were limited by the presumption that none of the patients with type 1 diabetes had ketoacidosis that might have affected lipid measurements. However, ketoacidosis is an acute condition that warrants hospitalisation, and such patients are unlikely to be recruited. In addition, our study design failed to classify the newly identified cases into different types of diabetes.

\section{What is already known on this subject?}

- Worldwide, over the past 20 years, the incidence of type 1 diabetes has almost doubled from $2.8 \%$ to $4.0 \%$ per year, while type 2 diabetes has increased 10 times in young children aged $6-12$ years of age and almost doubled among adolescents ranging from 7.3 to $13.9 / 100000$.

- However, there are limited numbers of community-based studies that have looked into the prevalence of type 1 and type 2 diabetes simultaneously among children and adolescents worldwide.

- Saudi Arabia, currently ranked seventh among the top 10 countries known for their high prevalence of diabetes globally, sets a good model to study the factors behind the increase in the prevalence of type 2 diabetes among children and adolescents.

\section{CONCLUSIONS}

Abnormal glucose metabolism is highly prevalent among Saudi children and adolescents, exceeding 10\%, and more than $90 \%$ of them were unaware of their disease. In this community, the prevalence of both known type 1 and type 2 diabetes was found to be higher than what had earlier been observed in other communities. It was also observed that age, male gender, high income and urban residency are important risk factors for diabetes and IFG among children and adolescents in this society. Other components of metabolic syndrome, namely obesity and

\section{What this study adds?}

- This study, as a part of the Saudi Abnormal Glucose Metabolism and Diabetes Impact Study (SAUDI-DM) explores the prevalence of type 1 and type 2 diabetes in addition to impaired fasting glucose and their modifiable and non-modifiable risk factors among children and adolescents.

- SAUDI-DM is a nationwide household population-based cross-sectional study using a multistage stratified cluster sampling technique taking into consideration the urban and rural ratio in the 13 administrative regions with the recruited participants being adjusted for age, area of residency and gender distribution to be compatible with the regional census. The STROBE guidelines were used for reporting the results of this study.

- The prevalence of both known type 1 and type 2 diabetes in children and adolescents was found to be higher than what had earlier been observed in other communities.

- Age, male gender, high income, urban residency and other components of metabolic syndrome, namely obesity and dyslipidaemia, are important risk factors for abnormal glucose metabolism among children and adolescents in this society.

- Abnormal glucose metabolism is highly prevalent among Saudi children and adolescents, exceeding 10\%, and more than $90 \%$ of them were unaware of their disease.

- There is an urgent need to adopt screening, prevention and early management programmes for children and adolescents. 
dyslipidaemia, represent important risks for abnormal glucose metabolism in this study. The findings from this study give a clear picture of the magnitude of type 1 and type 2 diabetes prevalence, especially in a society where more than $50 \%$ of its total population is younger than 20 years of age. There is an urgent need for adopting screening, prevention and early management programmes for children and adolescents in this society and similar other societies in the Middle East and globally, since these programmes have been proven to be effective.

Acknowledgements The author would like to acknowledge the team of the SAUDI-DM study and the staff at the University Diabetes Center for their contribution to the study, as well as the staff of the primary care centres from the Ministry of Health who were involved in this study.

Contributors KA designed the study, wrote the manuscript, designed the figures and interpreted the data, as well as approving the final version to be published.

Funding This study was funded by the University Diabetes Center at King Saud University, Ministry of Health and the Tawuniya Company for health insurance.

Competing interests None declared.

Ethics approval This study was approved by the Institutional Review Board (IRB) at the College of Medicine, King Saud University, Study number E-13-922.

Provenance and peer review Not commissioned; externally peer reviewed.

Open Access This is an Open Access article distributed in accordance with the Creative Commons Attribution Non Commercial (CC BY-NC 4.0) license, which permits others to distribute, remix, adapt, build upon this work non-commercially, and license their derivative works on different terms, provided the original work is properly cited and the use is non-commercial. See: http://creativecommons.org/ licenses/by-nc/4.0/

\section{REFERENCES}

1 Zimmet P, Alberti KG, Shaw J. Global and societal implications of the diabetes epidemic. Nature 2001;414:782-7.

2 Bloomgarden ZT. Type 2 diabetes in the young: the evolving epidemic. Diabetes Care 2004;27:998-1010.

3 Alberti G, Zimmet P, Shaw J, et al., Consensus Workshop Group. Type 2 diabetes in the young: the evolving epidemic: the international diabetes federation consensus workshop. Diabetes Care 2004;27:1798-811.

4 Matyka KA. Type 2 diabetes in childhood: epidemiological and clinical aspects. Br Med Bull 2008;86:59-75.

5 Amed S, Daneman D, Mahmud FH, et al. Type diabetes in children and adolescents. Expert Rev Cardiovasc Ther 2010;8:393-406.

6 Dabelea D, Mayer-Davis EJ, Saydah S, et al., SEARCH for Diabetes in Youth Study. Prevalence of type 1 and type 2 diabetes among children and adolescents from 2001 to 2009. JAMA 2014;311:1778-86.

7 Awa WL, Fach E, Krakow D, et al., DPV Initiative; German BMBF Competence Networks Diabetes mellitus and Obesity. Type 2 diabetes from pediatric to geriatric age: analysis of gender and obesity among 120,183 patients from the German/ Austrian DPV database. Eur J Endocrinol 2012;167:245-54.
8 Liu LL, Yi JP, Beyer J, et al., SEARCH for Diabetes in Youth Study Group. Type 1 and type 2 diabetes in Asian and Pacific Islander U.S. youth: the SEARCH for Diabetes in Youth Study. Diabetes Care 2009;32(Suppl 2):S133-40.

9 Copeland KC, Zeitler P, Geffner M, et al., TODAY Study Group. Characteristics of adolescents and youth with recent-onset type 2 diabetes: the TODAY cohort at baseline. J Clin Endocrinol Metab 2011;96:159-67.

10 Forsén T, Eriksson J, Tuomilehto J, et al. The fetal and childhood growth of persons who develop type 2 diabetes. Ann Intern Med 2000;133:176-82.

11 Al-Rubeaan K, Al-Manaa HA, Khoja TA, et al. Epidemiology of abnormal glucose metabolism in a country facing its epidemic: SAUDI-DM study. J Diabetes 2014

12 Barlow SE, Expert Committee. Expert committee recommendations regarding the prevention, assessment, and treatment of child and adolescent overweight and obesity: summary report. Pediatrics 2007;120(Suppl 4):S164-92.

13 American Diabetes Association. Screening for type 2 diabetes. Diabetes Care 2003;26(Suppl 1):S21-4.

14 [No authors listed]. National Cholesterol Education Program (NCEP): highlights of the report of the Expert Panel on Blood Cholesterol Levels in Children and Adolescents. Pediatrics 1992;89:495-501.

15 Doak CM, Visscher TLS, Renders CM, et al. The prevention of overweight and obesity in children and adolescents: a review of interventions and programmes. Obes Rev 2006;7:111-36.

16 Demmer RT, Zuk AM, Rosenbaum M, et al. Prevalence of diagnosed and undiagnosed type 2 diabetes mellitus among US adolescents: results from the continuous NHANES, 1999-2010. Am J Epidemio/ 2013:178:1106-13.

17 Nsiah-Kumi PA, Lasley S, Whiting M, et al. Diabetes, pre-diabetes and insulin resistance screening in Native American children and youth. Int J Obes (Lond) 2013;37:540-5

18 Li C, Ford ES, Zhao G, et al. Prevalence of pre-diabetes and its association with clustering of cardiometabolic risk factors and hyperinsulinemia among US adolescents: National Health and Nutrition Examination Survey 2005-2006. Diabetes Care 2009;32:342-7.

19 Dean HJ, Young TK, Flett B, et al. Screening for type-2 diabetes in aboriginal children in northern Canada. Lancet 1998:352:1523-4.

20 Wei JN, Sung FC, Lin CC, et al. National surveillance for type 2 diabetes mellitus in Taiwanese children. JAMA 2003:290:1345-50.

21 Caprio S, Plewe G, Diamond MP, et al. Increased insulin secretion in puberty: a compensatory response to reductions in insulin sensitivity. J Pediatr 1989;114:963-7.

22 Dabelea D, Hanson RL, Bennett PH, et al. Increasing prevalence of type II diabetes in American Indian children. Diabetologia 1998;41:904-10.

23 Barrett SC, Huffman FG, Johnson P, et al. A cross-sectional study of Jamaican adolescents' risk for type 2 diabetes and cardiovascular diseases. BMJ Open 2013;3:pii: e002817.

24 Mamtani R, Lowenfels $A B$, Sheikh J, et al. Adolescent prediabetes in a high-risk Middle East country: a cross-sectional study. JRSM Open 2014;5: 2054270414536550.

25 Glaser NS. Non-insulin-dependent diabetes mellitus in childhood and adolescence. PediatrClin North Am 1997;44:307-37.

26 Hamamy H, Antonarakis SE, Cavalli-Sforza LL, et al. Consanguineous marriages, pearls and perils: Geneva International Consanguinity Workshop Report. Genet Med 2011;13:841-7.

27 Sayeed M, Rhaman M, Fayzunnessa N, et al. Childhood diabetes in a Bangladeshi population. J Diabetes Mellitus 2013;3:33-7.

28 Franks PW, Hanson RL, Knowler WC, et al. Childhood predictors of young-onset type 2 diabetes. Diabetes 2007;56:2964-72 